\title{
Avaliação dos resultados iniciais do tratamento endovascular das lesões ateroscleróticas da artéria carótida interna (estudo retrospectivo)
}

\author{
Assesment of early outcomes of endovascular treatment \\ of atherosclerotic carotid lesions - a retrospective study
}

Eugênio Carlos de Almeida Tinoco*

O bjetivo: Analisar os resultados, nos primeiros 30 dias, da angioplastia com uso de stents sem proteção cerebral no tratamento das lesões ateroscleróticas da bifurcação carotídea.

Pacientes e métodos: Foi realizado um estudo retrospectivo de setembro de 1995 a 2001, no qual foram tratados 34 pacientes com 38 lesões envolvendo o bulbo carotídeo. O s pacientes foram acompanhados por um período de 30 dias, sendo o eco-color-D oppler o método deimagem utilizado para avaliar a perviedade do stent no primeiro dia após o procedimento, em todos os casos. Em relação à indicação, o procedimento foi realizado nas lesões maiores que $60 \%$ em pacientes sintomáticos e nas maiores que $70 \%$ nos assintomáticos, que correspondeu a $50 \%$ cada nesta amostra. T odos os pacientes seenquadravam em alto risco cirúrgico no que diz respeito à doença coronariana. 0 sexo masculino foi acometido em $62 \%$, e a idade variou de

\footnotetext{
Dissertação apresentada no curso de Pós-Graduação, nível Mestrado, área de Concentração Cirurgia Geral, Setor Cirurgia Vascular, do Departamento de Cirurgia da Faculdade de Medicina da Universidade Federal do Rio de Janeiro.

Orientador: Prof. Dr. Luis Felipe da Silva.

Apresentação:23/12/2004.

Banca examinadora: Prof. Dr. Gaudêncio Espinosa Lopez, Prof. Dr. Luis César Lopes da Silva e Prof. Dr. Antonio Luis Araújo.

Correspondência: Eugênio Carlos de Almeida Tinoco, Rua Pastor Abelar Suzano de Siqueira, 305/603, Cidade Nova, CEP 28300-000 Itaperuna, RJ. Tel.: (22) 3824.3299, Fax: (22) 3824.3507, E-mail: ecatinoco@globo.com.
}

Resumo submetido em 03.07.06, aceito em 21.08.06.
50 a 89 anos, com média de 69 anos. T odas as lesões eram ateroscleróticas, porém em cinco casos (13\%) se tratava de reestenose. $0 \mathrm{~s}$ fatores de risco principais foram: hiperten são arterial $(73,5 \%)$, diabetes $(14,7 \%)$ e tabagismo $(41,7 \%)$. T odos os procedimentos foram realizadoscom sucesso, sendo implantados, no total, 39 stents. Foram utilizadosstentsbalão eauto-expansíveis, havendo uma predominância dos últimos $(66,7 \%)$. $\mathrm{N}$ enhum dispositivo deproteção cerebral foi usado, por não haver disponibilidadeno mercado na época (19952001). Foi realizada arteriografia diagnóstica em todos os pacientes, para avaliar a possibilidade do tratamento endovascular, e, uma vez indicado, os pacientes iniciavam o uso deAAS $100 \mathrm{mg} /$ dia eticlopidina $500 \mathrm{mg} / \mathrm{dia}$, pelos menos 3 dias antes do procedimento, sendo mantidos até 30 dias após. Com exceção de um caso, a via de acesso femoral foi usada em todos os casos. Preferencialmente, foi realizado o implante primário do stent, reservando a pré-dilatação somente para os casos de estenose muito severa. A pós-dilatação foi real izada nos casos de stents auto-expansíveis.

Resultados: N esta série apresentada, houve um caso de AVC $(2,63 \%)$ que evoluiu para óbito $(2,63 \%)$, um caso $(2,63 \%)$ de infarto agudo do miocárdio e um de ataque isquêmico transitório.

C onclusão: N esta série de casos, a angioplastia carotídea com stent, mesmo sem proteção cerebral, associou-sea complicaçõessimilaresàs da en darterectomiacarotídea apresentadasnaliteratura, demonstrando ser potencial alternativa à endarterectomia no tratamento das lesões ateroscleróticas das carótidas, especial mente naquelas situações de risco cirúrgico maior. 